Kiyoshi Ichihara*, Ferruccio Ceriotti, Mori Kazuo, Yang-Yang Huang, Yoshihisa Shimizu, Haruki Suzuki, Masami Kitagawa, Kazuyoshi Yamauchi, Sadao Hayashi, Chia-Chun Tsou, Yoshikazu Yamamoto, Shigeo Ishida, Linda Leong, Michitaka Sano, Hwan Sub Lim, Akira Suwabe, Hee-Yeon Woo, Keiya Kojima, Yoshio Okubo on behalf of the Committee on Reference Intervals and Decision Limits, International Federation for Clinical Chemistry and Laboratory Medicine, and the Science Committee for the Asia-Pacific Federation of Clinical Biochemistry

\title{
The Asian project for collaborative derivation of reference intervals: (2) results of non- standardized analytes and transference of reference intervals to the participating laboratories on the basis of cross-comparison of test results
}

\begin{abstract}
Background: The 2009 Asian multicenter study for derivation of reference intervals (RIs) featured: 1) centralized measurements to exclude reagent-dependent variations; 2) inclusion of non-standardized analytes (hormones, tumor makers, etc.) in the target; and 3) cross-check of test results between the central and local laboratories. Transferability of centrally derived RIs for non-standardized analytes based on the cross-check was examined.

Methods: Forty non-standardized analytes were centrally measured in sera from 3541 reference individuals recruited by 63 laboratories. Forty-four laboratories collaborated in the cross-check study by locally measuring aliquots of sera from 9 to 73 volunteers (average 22.2). Linear relationships were obtained by the reduced major-axis regression. Error in converting RIs using the regression line was expressed by the coefficient of variation of slope $b[\mathrm{CV}(b)]$. $\mathrm{CV}(\mathrm{b})<10 \%$ was set as the cut-off value allowing the conversion. The significance of factors for partitioning RIs was determined similarly as in the first report.

Results: Significant sex-, age-, and region-related changes in test results were observed in 17,15 , and 11 of the 40 analytes, respectively. In the cross-comparison study, test results were not harmonized in the majority of immunologically measured analytes, but their average $\mathrm{CV}(\mathrm{b}) \mathrm{s}$ were $<10 \%$ except for total protein, cystatin C, CA19-9, free thyroxine, and triiodothyronine. After conversion, $74 \%$ of centrally derived RIs were transferred to each local laboratory.
\end{abstract}

Conclusions: Our results point to the feasibility of: 1) harmonizing test results across different laboratories; and 2) sharing centrally derived RIs of non-standardized analytes by means of comparative measurement of a set of commutable specimens.

Keywords: harmonization; immunoassays; multicenter study; standardization.

*Corresponding author: Kiyoshi Ichihara, MD, PhD, Faculty of Health Sciences, Department of Clinical Laboratory Sciences, Yamaguchi University Graduate School of Medicine, MinamiKogushi 1-1-1, Ube 755-8505, Japan, Phone: +81 836222884 , Fax: +81 836 355213, E-mail: ichihara@yamaguchi-u.ac.jp Ferruccio Ceriotti: Diagnostica e Ricerca San Raffaele, San Raffaele Scientific Institute Hospital, Milan, Italy

Mori Kazuo: Beckman Coulter Japan Co., Tokyo, Japan

Yang-Yang Huang: Laboratory Medicine Department, Cathay General Hospital, Taipei, Taiwan

Yoshihisa Shimizu: Faculty of Health Sciences, Department of Clinical Laboratory Sciences, Yamaguchi University Graduate School of Medicine, Ube, Japan

Haruki Suzuki: Clinical Laboratory, Hokkaido University Hospital, Sapporo, Japan

Masami Kitagawa: Clinical Laboratory, Yoshida Hospital, Asahikawa, Japan

Kazuyoshi Yamauchi: Molecular Clinical Pathology, University of Tsukuba, Graduate School of Comprehensive Human Sciences, Tsukuba, Japan

Sadao Hayashi: Laboratory for Clinical Investigation, Osaka University Hospital, Suita, Japan 
Chia-Chun Tsou: Clinical Laboratory, Mackay Memorial Hospital, Taipei, Taiwan

Yoshikazu Yamamoto: Department of Clinical Pathology, Tenri Hospital, Tenri, Japan

Shigeo Ishida: Clinical Laboratory, Osaka Municipal University Hospital, Osaka, Japan

Linda Leong: Clinical Laboratory, Gleneagles Intan Medical Centre, Kuala Lumpur, Malaysia

Michitaka Sano: Clinical Laboratory, National Cerebral and

Cardiovascular Center Hospital, Suita, Japan

Hwan Sub Lim: Department of Laboratory Medicine, Myongji Hospital, Kwandong University College of Medicine, Kyunggi, Korea Akira Suwabe: Department of Laboratory Medicine, Iwate Medical School Hospital, Morioka, Japan

Hee-Yeon Woo: Department of Laboratory Medicine, Kangbuk Samsung Hospital, Sung Kyun Kwan University, Seoul, Korea Keiya Kojima: Clinical Laboratory, Hirosaki University Hospital, Hirosaki, Japan

Yoshio Okubo: Clinical Laboratory, Tokyo University Hospital, Tokyo, Japan

\section{Non-standard abbreviations}

ADP, adiponectin; AFP, $\alpha$-fetoprotein; ApoA1, apolipoprotein A-I; ApoB, apolipoprotein B; ApoE, apolipoprotein E; ASO, anti-streptolysin O; BAP, bone alkaline phosphatase; BC, Beckman Coulter; CA15-3, carbohydrate antigen 15-3; CA19-9, carbohydrate antigen 19-9; CA125, carbohydrate antigen 125; CEA, carcinoembryonic antigen; CV(b), coefficient of variation of slope 'b'; CysC, cystatin C; DHEA-S, dehydroepiandrosterone sulfate; EPO, erythropoietin; Fe, iron; Ferritin, ferritin; Folate, folic acid; FSH, follicle-stimulating hormone; FT3, free triiodothyronine; FT4, free thyroxine; IgE, immunoglobulin E; IP, inorganic phosphate; JSCC, Japan Society of Clinical Chemistry; LH, luteinizing hormone; LIP, lipase; LL, lower limit; Lp(a), lipoprotein (a); PG1, pepsinogen I; PG2, pepsinogen II; PRL, prolactin; PSA, prostate-specific antigen; PTH, intact parathyroid hormone; RBP, retinol-binding protein; RI, reference interval; SAA, serum amyloid A; SE, standard error; sTf-R, soluble transferrin receptor; TG, thyroglobulin; TP, protein, total; TRAP-5b, tartrate-resistant acid phosphatase 5b; TSH, thyroid-stimulating hormone; UIBC, unsaturated ironbinding capacity; UL, upper limit; VitB12, vitamin B12.

\section{Introduction}

There has been growing demand for the conduction of a multicenter study to derive reference intervals (RIs) of commonly ordered laboratory tests that have been globally standardized. The Asian multicenter study conducted in 2009 was unique in that it also attempted to derive common RIs for non-standardized analytes, such as protein hormones and tumor markers, most of which were measured by immunoassays [1]. The key strategy was to centralize measurement by transporting all serum specimens in a deep-frozen state to central laboratories located in Japan. A single reagent and platform for each of the analytes was used to detect regionality of test results without assay-related variations.

The policy was appropriate for the standardized analytes such as creatinine, uric acid, total cholesterol, and electrolytes. However, for the non-standardized analytes, each RI determined was only relevant to the laboratories that used the same reagents as the central laboratory. Therefore, a scheme was implemented to cross-compare test results between the central and collaborating laboratories. For this purpose, parts of the frozen aliquots of sera remained at each laboratory and were measured locally. We: 1) analyzed sex-, age-, and region-related changes in test results for 40 non-standardized analytes; 2) derived their RIs for the central laboratory; and 3) evaluated the transferability of the RIs to each collaborating laboratory on the basis of cross-check testing.

\section{Materials and methods}

\section{Multicenter study conducted in East and Southeast Asian}

The framework of the Asian multicenter study was described in the companion paper, Part 1 of this article [1]. In brief, 48 laboratories from 14 cities distributed widely from the north to the south of Japan, and 15 laboratories in nine cities outside Japan (Seoul, Beijing, Taipei, Tainan, Hong Kong, Macau, Ho Chi Minh City, Kuala Lumpur, and Jakarta) took part in the study. A total of 3541 healthy individuals (2082 from Japan and 1459 from outside Japan) between 20 and 64 years of age were recruited using common criteria, and samples of their blood were drawn at basal conditions. After a selection process for excluding those with apparent abnormal results in basic screening test items such as TG, HDL-C, glucose, etc., was performed, test results from a total of 3314 subjects (1438 males, 1876 females) were served for data analyses. Names, abbreviations, assay methods, and imprecision of the analytes for which test results were evaluated in this report are listed in Table 1.

\section{Collection and measurement of specimens for cross-check testing}

The standard amount of blood taken from each volunteer was $17 \mathrm{~mL}$ $(8.5 \mathrm{~mL} /$ tube $\times 2)$. The tubes, Vacutainer SST (capacity: $8.5 \mathrm{~mL}$ ) containing a clot-activator, were provided by Becton-Dickinson Corp. (Franklin Lakes, NJ, USA). Sera were separated into CryoTube cryovials 
Table 1 List of assay methods and assay imprecision near the mid-normal range.

\begin{tabular}{|c|c|c|c|c|c|c|}
\hline Abbr & Analytes & Method & Reagent & Analyzer & $\begin{array}{r}\text { Within- } \\
\text { day CV, \% }\end{array}$ & $\begin{array}{l}\text { Between- } \\
\text { day CV, \% }\end{array}$ \\
\hline TP & Protein, total & Timed endpoint biuret method & $\mathrm{BC}$ & (1) & 0.76 & 1.49 \\
\hline CysC & Cystatin C & Latex immunoturbidimetric method & NM & (1) & 1.54 & 2.56 \\
\hline IP & Inorganic phosphate & Timed endpoint molybdate UV method & $\mathrm{BC}$ & (1) & 1.55 & 0.00 \\
\hline $\mathrm{Fe}$ & Iron & Timed endpoint direct colorimetry & $\mathrm{BC}$ & (1) & 1.97 & 1.46 \\
\hline UIBC & Unsaturated iron-binding capacity & Timed endpoint direct colorimetry & $\mathrm{BC}$ & (1) & 1.74 & 2.18 \\
\hline Apo A1 & Apolioprotein A-I & Turbidimetric method & $\mathrm{BC}$ & (1) & 1.91 & 3.72 \\
\hline Apo B & Apolioprotein B & Turbidimetric method & $\mathrm{BC}$ & (1) & 1.56 & 2.59 \\
\hline Apo $\mathrm{E}$ & Apolioprotein E & Turbidimetric immunoassay & NM & (1) & 1.62 & 2.71 \\
\hline $\operatorname{Lp}(\mathrm{a})$ & Lipoprotein (a) & Turbidimetric immunoassay & NM & (1) & 1.74 & 3.03 \\
\hline LIP & Lipase & Enzymatic rate method & $\mathrm{BC}$ & (1) & 2.36 & 3.43 \\
\hline $\lg \mathrm{E}$ & Immunoglobulin E & Chemiluminescent enzyme immunoassay & $\mathrm{BC}$ & $(2)$ & 2.61 & 2.17 \\
\hline SAA & Serum amyloid $A$ & Latex immunoturbidimetric method & EM & (1) & 1.64 & 2.63 \\
\hline RBP & Retinol-binding protein & Latex immunoturbidimetric method & NM & (1) & 1.78 & 3.20 \\
\hline ASO & Anti-streptolysin 0 & Turbidimetric immunoassay & $\mathrm{BC}$ & (1) & 1.83 & 3.34 \\
\hline sTf-R & Soluble transferin receptor & Chemiluminescent enzyme immunoassay & $\mathrm{BC}$ & $(2)$ & 1.30 & 1.50 \\
\hline Ferritin & Ferritin & Chemiluminescent enzyme immunoassay & $\mathrm{BC}$ & $(2)$ & 1.79 & 2.01 \\
\hline EPO & Erythropoiein & Chemiluminescent enzyme immunoassay & $\mathrm{BC}$ & $(2)$ & 1.74 & 1.76 \\
\hline VitB12 & Vitamin B12 & Chemiluminescent enzyme immunoassay & $\mathrm{BC}$ & $(2)$ & 3.33 & 2.36 \\
\hline Folate & Folic acid & Chemiluminescent enzyme immunoassay & $\mathrm{BC}$ & $(2)$ & 1.68 & 0.77 \\
\hline AFP & $\alpha$-fetotein & Chemiluminescent enzyme immunoassay & $\mathrm{BC}$ & $(2)$ & 2.00 & 1.90 \\
\hline CEA & Carcinoembryonic antigen & Chemiluminescent enzyme immunoassay & $\mathrm{BC}$ & $(2)$ & 3.03 & 2.49 \\
\hline CA19-9 & Carbohydrate antigen $19-9$ & Chemiluminescent enzyme immunoassay & $\mathrm{BC}$ & $(2)$ & 3.44 & 5.46 \\
\hline CA15-3 & Carbohydrate antigen 15-3 & Chemiluminescent enzyme immunoassay & $\mathrm{BC}$ & $(2)$ & 1.94 & 2.51 \\
\hline CA125 & Carbohydrate antigen 125 & Chemiluminescent enzyme immunoassay & $\mathrm{BC}$ & $(2)$ & 2.57 & 2.16 \\
\hline PSA & Prostate-specific antigen & Chemiluminescent enzyme immunoassay & $\mathrm{BC}$ & $(2)$ & 2.54 & 3.03 \\
\hline PG1 & Pepsinogen I & Latex immunoturbidimetric method & EM & (3) & 1.61 & 2.70 \\
\hline PG2 & Pepsinogen II & Latex immunoturbidimetric method & EM & (3) & 1.55 & 2.58 \\
\hline DHEA-S & Dehydroepiandrosterone sulfate & Chemiluminescent enzyme immunoassay & $\mathrm{BC}$ & $(2)$ & 1.52 & 1.46 \\
\hline PRL & Prolactin & Chemiluminescent enzyme immunoassay & $\mathrm{BC}$ & $(2)$ & 1.16 & 0.98 \\
\hline $\mathrm{LH}$ & Luteinizing hormone & Chemiluminescent enzyme immunoassay & $\mathrm{BC}$ & $(2)$ & 2.64 & 1.95 \\
\hline FSH & Follicle-stimulating hormone & Chemiluminescent enzyme immunoassay & $\mathrm{BC}$ & $(2)$ & 2.68 & 1.80 \\
\hline TSH & Thyroid-stimulating hormone & Chemiluminescent enzyme immunoassay & $\mathrm{BC}$ & $(2)$ & 2.23 & 1.53 \\
\hline FT4 & Free thyroxine & Chemiluminescent enzyme immunoassay & $\mathrm{BC}$ & $(2)$ & 2.07 & 2.14 \\
\hline FT3 & Free triiodothyronine & Chemiluminescent enzyme immunoassay & $\mathrm{BC}$ & $(2)$ & 1.95 & 1.05 \\
\hline $\operatorname{Tg}$ & Thyroglobulin & Chemiluminescent enzyme immunoassay & $\mathrm{BC}$ & $(2)$ & 1.95 & 1.88 \\
\hline Insulin & Insulin & Chemiluminescent enzyme immunoassay & $\mathrm{BC}$ & $(2)$ & 1.75 & 2.06 \\
\hline ADP & Adiponectin & Latex immunoturbidimetric method & $\mathrm{OP}$ & (3) & 1.64 & 2.76 \\
\hline PTH & Intact parathyroid hormone & Chemiluminescent enzyme immunoassay & $\mathrm{BC}$ & $(2)$ & 1.92 & 1.76 \\
\hline BAP & Bone alkaline phosphatase & Chemiluminescent enzyme immunoassay & $\mathrm{BC}$ & $(2)$ & 1.85 & 1.98 \\
\hline TRAP- $5 b$ & $\begin{array}{l}\text { Tartrate-resistant acid } \\
\text { phosphatase } 5 b\end{array}$ & Enzyme immunoassay & NM & (4) & 1.62 & 2.71 \\
\hline
\end{tabular}

BC, Beckman Coulter; EM, Eiken Medical; NM, Nitobo Medical; OP, Otsuka Pharma. (1) UniCell DxC, BC, Japan (2) UniCell DxI, BC, Japan (3) JCA-BM 6050, JEOL Ltd, Japan (4) Manual.

(capacity: $4.8 \mathrm{~mL}$ ) (Nunc, Roskilde, Denmark), and put into deep freezer immediately as described in the first part of this paper.

Among the 63 collaborating laboratories, 44 voluntarily took part in the exercise of cross-check testing for secondary transference of RIs. Those volunteers who agreed to cooperate in the cross-check testing scheme gave one additional tube of blood $(8.5 \mathrm{~mL})$. These additional specimens were processed and stored identically with the other specimens to be transported to the central laboratory in Japan. The number of specimens used for the cross-check ranged from 9 to 73 (average 22.2). A total of 65 analytes, including standardized ana- lytes for which RIs and related data analysis were reported [1], were available for the cross-comparison of test results.

The frozen specimens stored at $-80^{\circ} \mathrm{C}$ were thawed and measured identically with specimens for routine assay under well-controlled condition during the period when the collective measurements were being conducted. The test results recorded in an Excel file together with assay methods, reagents, and assay platforms were sent to the Secretariat of this study located at the Ichihara Research Laboratory in the Yamaguchi University Graduate School of Medicine, Ube, Japan. 


\section{Quality control of the assays}

Quality control (QC) monitoring in the central laboratory was carried out chiefly by use of a set of four fresh-frozen pooled sera from healthy individuals, stored in aliquots at $-80^{\circ} \mathrm{C}$. Additional seven sets of commercially available QC specimens with artificially adjusted matrix were also measured as a supplemental monitoring procedure (Supplementary Table 1, which accompanies the article at http://www.degruyter.com/ view/j/cclm.2013.51.issue-7/issue-files/cclm.2013.51.issue-7.xml). Each set of QC specimens consisted of two to three levels (below, within, or above the normal level). Two sets were for chemically measured tests and the other five were for the immunologically measured tests. The same sets of specimens were also distributed to the local laboratories that took part in the cross-check study. The test results of commercial QC sera were reported together with those of specimens for the cross check.

\section{Statistical analysis}

Analysis of sources of variation and derivation of RIs were performed in the exact same way as described in Part 1 of this paper [1]. The linear structural relation of test results between the central laboratory and each local laboratory was determined by use of the major axis regression method [2-4], expressed as follows with $\bar{x}$ and $\bar{y}$ and $b$ denoting the averages of $x$ (test result from the central laboratory) and $y$ (corresponding result from a local laboratory), and the slope, respectively.

$$
(y-\bar{y})=b \times(x-\bar{x})
$$

The slope $b$ is determined by the following formula:

$$
\begin{gathered}
b=\sqrt{\frac{S_{y y}}{S_{x x}}} \\
S_{x x}=\sum_{i=1}^{n}\left(x_{i}-\bar{x}\right)^{2} \quad S_{y y}=\sum_{i=1}^{n}\left(y_{i}-\bar{y}\right)^{2}
\end{gathered}
$$

where $n, S_{x x}$, and $S_{y y}$ denote the sample size, and sums of squareddeviations of values from each of the central and local laboratories, respectively.

Using the regression Equation (1), the lower limit (LL) and upper limit (UL) of a RI centrally derived can be converted to those of a local laboratory (LL' and UL') by use of the following formulae.

$$
\begin{aligned}
& \mathrm{LL}^{\prime}=\bar{y}+b \times(L L-\bar{x}) \\
& \mathrm{UL}^{\prime}=\bar{y}+b \times(U L-\bar{x})
\end{aligned}
$$

The standard error of the slope $b, \operatorname{SE}(\mathrm{b})$, was determined by the bootstrap method by re-sampling the same number of data from the original dataset [5]. Mathematical derivation of $\mathrm{SE}(\mathrm{b})$ is known to be difficult but it is said to be approximated by the standard error of the slope $b^{\prime}, \mathrm{SE}\left(\mathrm{b}^{\prime}\right)$, computed by the ordinary least square method using the following formula $(2,4)$ (see Appendix 1).

$$
\begin{gathered}
y-\bar{y}=b^{\prime}(x-\bar{x}) \\
b^{\prime}=\frac{S x y}{S x x}, \quad S_{x y}=\sum_{i=1}^{n}\left(x_{i}-\bar{x}\right)\left(y_{i}-\bar{y}\right)
\end{gathered}
$$

$$
S E(b) \cong S E\left(b^{\prime}\right)=\sqrt{\frac{\left(1-r^{2}\right) S_{y y}}{(n-2) S_{x x}}}=b \sqrt{\frac{1-r^{2}}{n-2}}
$$

For standardized comparison of the $\mathrm{SE}(\mathrm{b})$, it was expressed as the coefficient of variation of slope $b[\mathrm{CV}(\mathrm{b})]$ by taking its ratio to ' $b$ ' as follows:

$$
C V(b)=\frac{S E(b)}{b} \times 100 \simeq 100 \sqrt{\frac{1-r^{2}}{n-2}}
$$

$\mathrm{CV}$ (b) was used as a guide to judge convertibility of the RIs derived from the central laboratory to those of the participating laboratories. Although many factors have to be considered in deciding when to allow the conversion, as explained in the Discussion, we empirically set $\mathrm{CV}(\mathrm{b})=10 \%$ as the cut-off value for the conversion.

\section{Results}

\section{Sources of variations of test results}

The magnitudes of sex-, age-, and region-specific differences in test results examined by three-level nested ANOVA are as shown in Table 2. We regarded a SD ratio (SDR) of $\geq 0.3$ of any given factor as significant for consideration of partitioning RIs by the factor, as we did in Part 1 of this paper. With regard to between-sex difference, we compared the judgment by SDR for sex [SDR-sex] (third rightmost column in Table 2) with that by the Harris-Boyd method employing the adjusted $\mathrm{z}$ score [az] (fourth rightmost column in Table 2), whose critical limit is usually set as 3.0. The two approaches matched quite well. Significant sex-related change was identified by az score and SDR-sex in 20 and 17 of 40 analytes, respectively, including prominent examples of RBP, ferritin, and PSA. Similarly, significant between-region differences were found by SDR for between-region (SDR-reg) in nine analytes, including folate, CA15-3, adiponectin, and PTH as notable examples (Figure 1). Similar figures are provided for all the analytes in Supplemental Figure 1. Age-related changes were noted in 15 analytes including ApoB, DHEA-S, LH, and FSH. As the magnitude of between-region and -age differences often differ between the two sexes, we performed three-level nested ANOVA after separating the dataset by sex and introducing BMI as a third factor for the analysis (Supplementary Table 2). The two values shown within the parenthesis in the last two columns in Table 2 represent corresponding SDR-reg and -age values for males and females, respectively. We observed unmatched region- and age-related changes between the two sexes in three analytes (CysC, ferritin, and TRAP-5b) and seven 


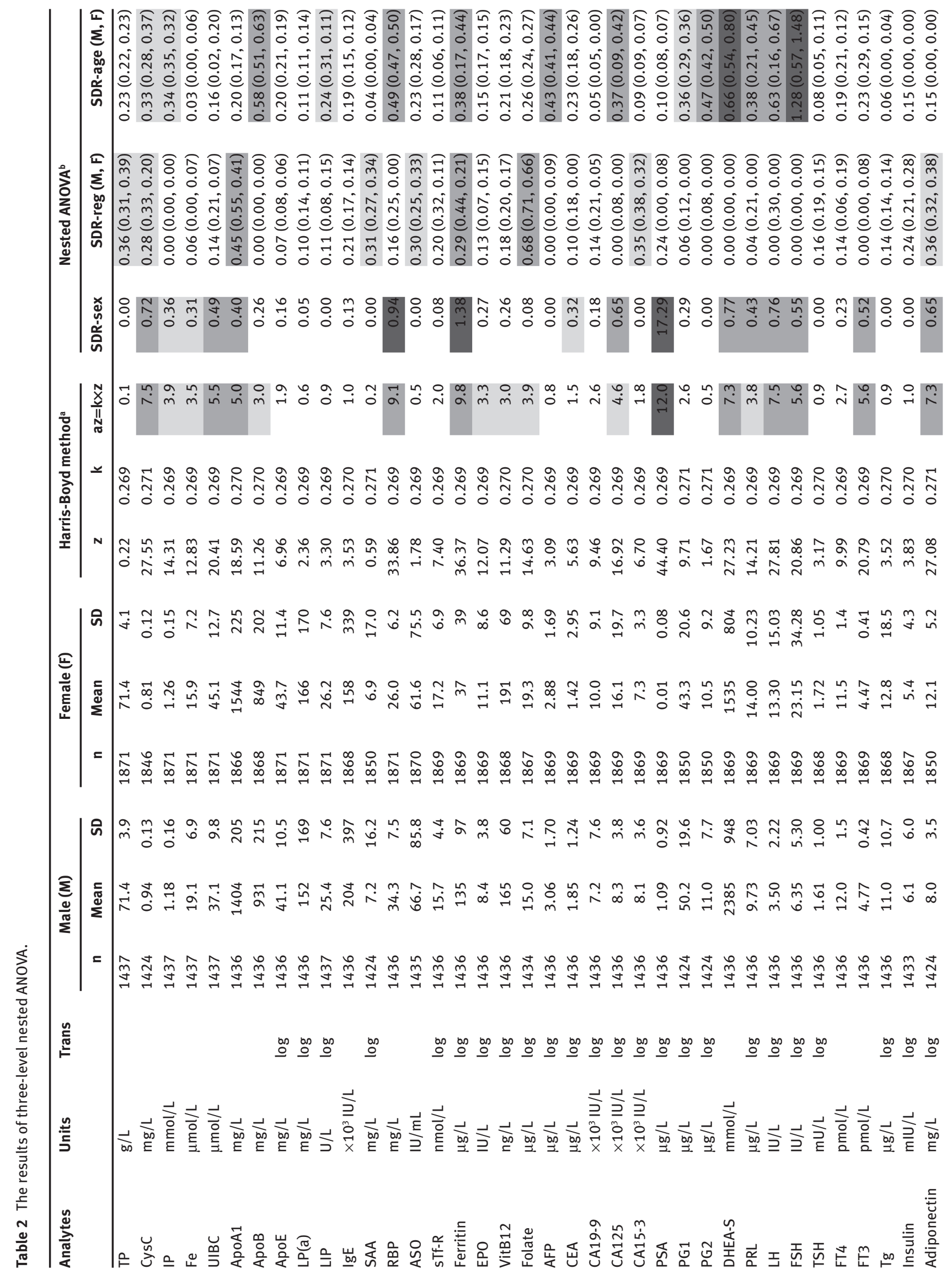




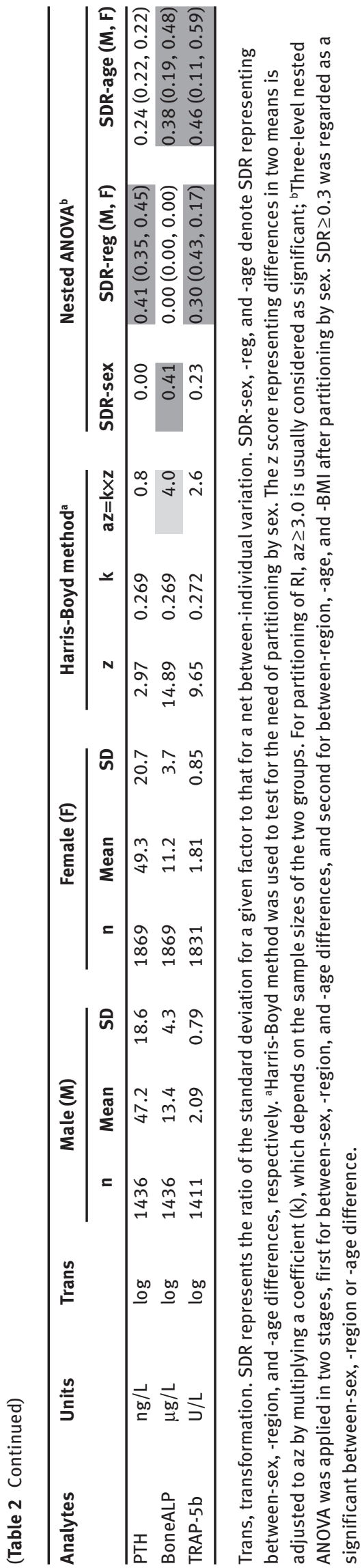

analytes (LIP, CA125, PRL, LH, FSH, BoneALP, and TRAP$5 b)$, respectively.

\section{Reference intervals}

The RIs derived by the same procedures as described in Part 1 of this paper are listed in Table 3, and in Supplementary Table 3 for full results for region-specific RIs. The age-specific RIs derived for each sex and decade of age are listed in Supplementary Table 4.

\section{Assay reagents or platforms involved in the cross-check testing}

In this report, those laboratories using $<15$ specimens for the comparison were omitted due to lack of adequate statistical power in the evaluation. Thus, the actual datasets used for the evaluation were reduced to those from 37 of the original 44 laboratories. Furthermore, those analytes with participation of less than eight laboratories for crosscheck testing were also omitted, thus reducing the number of analytes actually evaluated to 22 from the original 34 .

The combinations of reagents and assay platforms actually used for the measurements by local laboratories were so divergent that we did not list them here. However, those analytes measured by immunoassays could be subgrouped by manufacturers of assay reagents/platforms into a small number of categories as listed in Table 4 with Roche, Abbott, and Tosoh accounting for the majority of the immunoassays.

\section{Typical two-dimensional scattergrams for the cross-check testing}

Typical cross-check results for four non-standardized analytes (AFP, CA19-9, PSA, and TSH) are illustrated in Figure 2. The x-axis represents test results by the central laboratory, and the y-axis represents results obtained at three local laboratories (Laboratories 1104, 1501, and 4103). These results were chosen to demonstrate representative results, and their reagents and assay platforms differed from those of the central laboratory. The comparisons of the values were made based on conventional units because the figures are just meant to demonstrate comparability of the values.

CV(b)s shown in the left top corner of the graph inversely correlated with the correlation coefficients shown in the right bottom. However, it should be noted 

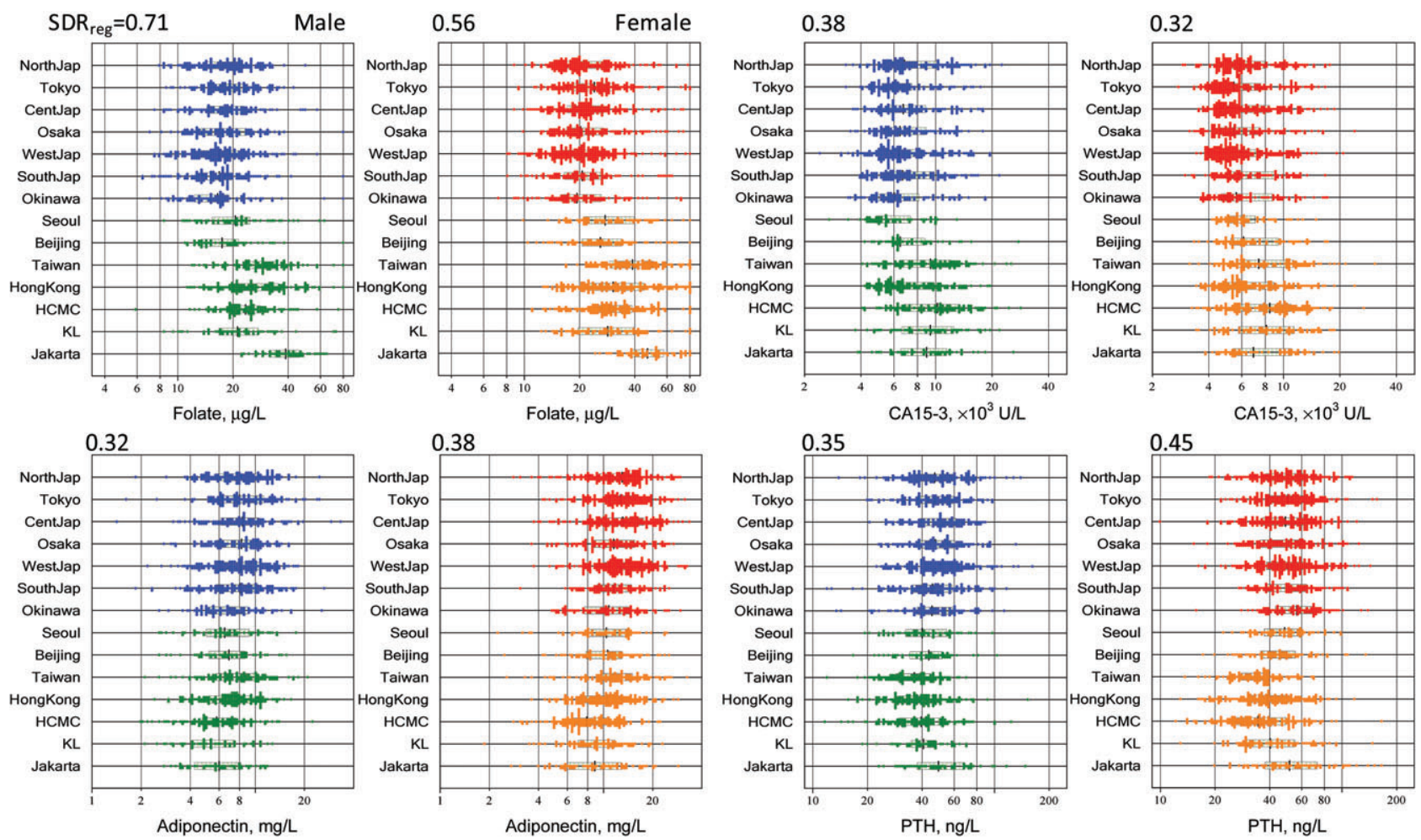

Figure 1 Regionality of test results observed in four analytes.

Each panel consists of two figures, one for males and one for females. Test results were sub-grouped into 14 areas: seven from Japan (blue, male; red, female) and another seven from East and Southeast Asia (green, male; orange, female). The regions were aligned from the north to the south. The box within the scattergrams represents the central $50 \%$ range, and the vertical line in the middle of the box indicates the median.

that $\mathrm{CV}(\mathrm{b})$ decreases proportionately with the square root of the sample size used for the linear regression as expected from Equation (6). As described below, the crosscomparison for AFP, PSA, and TSH generally resulted in close correlations, although regression lines mostly did not match with the line of concordance $(\mathrm{y}=\mathrm{x})$. However, the graphs for CA19-9 showed a large scatter around regression lines with larger $\mathrm{CV}(\mathrm{b}) \mathrm{s}$.

\section{The CV(b) list}

The list of $\mathrm{CV}(\mathrm{b})$ computed from the regression line for the cross-check between the central laboratory and each local laboratory is shown in Table 5. The first column represents the sequence number of the local laboratories listed. The second column represents the number of samples actually measured by each local laboratory. Cells are colored according to the magnitude of $\mathrm{CV}(\mathrm{b})$. The average $\mathrm{CV}(\mathrm{b})$ and the percentage of laboratories that received the transference service of RIs with $\mathrm{CV}(\mathrm{b})<10 \%$ are shown in the two bottom rows. A high rate of transferability of RI ( $>80 \%)$ was attained among the participating laboratories in the vast majority of analytes. Notable exceptions were those for TP, CysC, CA19-9, FT4, and FT3, which showed $<80 \%$ transferability. The list of $\mathrm{CV}(\mathrm{b})$ for the standardized analytes are shown in Supplementary Table 5. Their CV(b) were generally low except for Alb, Na, K, Cl, and Ca, with a CV(b) exceeding $10 \%$ in most of the cases.

\section{Commutability of commercial QC specimens in the cross-comparison}

We had expected that commercial QC specimens would give much the same results in the cross-comparison of test results between the central and local laboratories. However, prominent differences were observed with the use of the QC specimens in test results for chloride, inorganic phosphate, HDL-C, LDL-C, amylase, and, partly, AST, ALP, sodium, potassium, and total protein. Figure 3 shows typical results of the cross-comparison plotted for two datasets: one for individual sera (black dots), as in 
Table 3 List of RIs derived.

\begin{tabular}{|c|c|c|c|c|c|c|c|c|c|c|c|c|c|c|c|}
\hline \multicolumn{4}{|c|}{ International unit } & \multirow[t]{2}{*}{$\mathrm{n}$} & \multicolumn{3}{|c|}{ Male + Female } & \multirow[t]{2}{*}{$n$} & \multicolumn{3}{|c|}{ Male } & \multirow[t]{2}{*}{$n$} & \multicolumn{3}{|c|}{ Female } \\
\hline Analytes & Units & SDR-reg & Area & & LL & Me & UL & & $\mathbf{L L}$ & Me & $\overline{U L}$ & & $\overline{L L}$ & $\mathrm{Me}$ & UL \\
\hline \multirow[t]{4}{*}{ TP } & \multirow[t]{4}{*}{$\mathrm{g} / \mathrm{L}$} & \multirow[t]{4}{*}{0.36} & Asia All & 2891 & 64 & 71 & 79 & 1279 & 64 & 71 & 79 & 1611 & 64 & 71 & 19 \\
\hline & & & Japanese & 1732 & 64 & 71 & 78 & 784 & 64 & 71 & 78 & 948 & 63 & 71 & 78 \\
\hline & & & Chinese & 601 & 64 & 71 & 79 & 266 & 65 & 71 & 78 & 334 & 64 & 71 & 80 \\
\hline & & & SE Asia & 479 & 66 & 74 & 82 & 196 & 66 & 74 & 82 & 283 & 66 & 74 & 82 \\
\hline \multirow[t]{4}{*}{ CysC } & \multirow[t]{4}{*}{$\mathrm{mg} / \mathrm{L}$} & \multirow[t]{4}{*}{0.28} & Asia All & 2884 & 0.63 & 0.85 & 1.16 & 1274 & 0.71 & 0.92 & 1.21 & 1605 & 0.61 & 0.80 & 1.05 \\
\hline & & & Japanese & 1725 & 0.62 & 0.83 & 1.12 & 782 & 0.69 & 0.90 & 1.17 & 944 & 0.61 & 0.78 & 1.03 \\
\hline & & & Chinese & 600 & 0.65 & 0.87 & 1.22 & 267 & 0.74 & 0.95 & 1.31 & 331 & 0.62 & 0.80 & 1.05 \\
\hline & & & SE Asia & 477 & 0.65 & 0.90 & 1.21 & 196 & 0.79 & 0.98 & 1.29 & 282 & 0.63 & 0.84 & 1.10 \\
\hline IP & $\mathrm{mmol} / \mathrm{L}$ & 0.00 & Asia All & 2889 & 0.93 & 1.22 & 1.54 & 1278 & 0.88 & 1.18 & 1.50 & 1610 & 0.98 & 1.26 & 1.55 \\
\hline $\mathrm{Fe}$ & $\mathrm{mmol} / \mathrm{L}$ & 0.06 & Asia All & 2888 & 5 & 17 & 33 & 1271 & 8 & 18 & 34 & 1609 & 4 & 15 & 32 \\
\hline UIBC & $\mathrm{mmol} / \mathrm{L}$ & 0.14 & Asia All & 2889 & 21 & 40 & 69 & 1276 & 19 & 37 & 55 & 1612 & 24 & 44 & 75 \\
\hline ApoA1 & $\mathrm{mg} / \mathrm{L}$ & 0.45 & Asia All & 2883 & 1112 & 1462 & 1967 & 1275 & 1072 & 1383 & 1839 & 1606 & 1173 & 1531 & 2000 \\
\hline & & & Japanese & 1724 & 1175 & 1513 & 2017 & 778 & 1144 & 1442 & 1913 & 943 & 1240 & 1574 & 2052 \\
\hline & & & Chinese & 598 & 1089 & 1399 & 1878 & 265 & 1036 & 1323 & 1628 & 333 & 1150 & 1484 & 1968 \\
\hline & & & SE Asia & 471 & 1078 & 1361 & 1864 & 195 & 1012 & 1266 & 1585 & 283 & 1108 & 1435 & 1922 \\
\hline ApoB & $\mathrm{mg} / \mathrm{L}$ & 0.00 & Asia All & 2884 & 541 & 849 & 1329 & 1278 & 570 & 908 & 1371 & 1608 & 522 & 810 & 1256 \\
\hline ApoE & $\mathrm{mg} / \mathrm{L}$ & 0.07 & Asia All & 2872 & 25 & 41 & 64 & 1275 & 25 & 39 & 62 & 1602 & 26 & 42 & 66 \\
\hline $\mathrm{LP}(\mathrm{a})$ & $\mathrm{mg} / \mathrm{L}$ & 0.10 & Asia All & 2891 & 2 & 99 & 689 & 1278 & 1 & 90 & 692 & 1612 & 3 & 108 & 648 \\
\hline LIP & $\mathrm{U} / \mathrm{L}$ & 0.11 & Asia All & 2875 & 13 & 24 & 39 & 1270 & 13 & 23 & 41 & 1595 & 14 & 25 & נ \\
\hline $\lg E$ & $\times 10^{3} \mathrm{IU} / \mathrm{L}$ & 0.21 & Asia All & 1259 & 2 & 42 & 528 & 559 & 3 & 61 & 633 & 700 & 2 & 33 & 390 \\
\hline SAA & $\mathrm{mg} / \mathrm{L}$ & 0.31 & Asia All & 2846 & 1.0 & 3.8 & 20.4 & 1257 & 1.1 & 3.7 & 23.5 & 1589 & 1.0 & 3.9 & 18.3 \\
\hline & & & Japanese & 1698 & 0.9 & 3.2 & 16.4 & 767 & 0.9 & 3.3 & 16.4 & 930 & 1.0 & 3.2 & 15.9 \\
\hline & & & Chinese & 595 & 1.4 & 4.8 & 22.9 & 265 & 1.3 & 4.7 & 27.8 & 331 & 1.4 & 4.9 & 21.3 \\
\hline & & & SE Asia & 477 & 1.4 & 5.7 & 24.7 & 193 & 1.4 & 5.5 & 22.3 & 281 & 1.6 & 5.7 & 25.2 \\
\hline RBP & $\mathrm{mg} / \mathrm{L}$ & 0.16 & Asia All & 2887 & 17.4 & 28.2 & 47.5 & 1273 & 21.9 & 33.4 & 49.7 & 1604 & 17.3 & 24.5 & 40.3 \\
\hline ASO & $\times 10^{3} \mathrm{IU} / \mathrm{L}$ & 0.30 & Asia All & 2879 & 2 & 37 & 293 & 1272 & 3 & 37 & 315 & 1611 & 1 & 39 & 248 \\
\hline & & & Japanese & 1730 & 1 & 38 & 292 & 778 & 2 & 37 & 343 & 946 & 2 & 36 & 295 \\
\hline & & & Chinese & 592 & 1 & 27 & 159 & 264 & 2 & 29 & 193 & 334 & 1 & 26 & 163 \\
\hline & & & SE Asia & 477 & 10 & 60 & 332 & 195 & 8 & 61 & 316 & 281 & 11 & 63 & 308 \\
\hline STf-R & $\mathrm{nmol} / \mathrm{L}$ & 0.20 & Asia All & 2853 & 10.2 & 15.0 & 28.3 & 1262 & 10.4 & 14.9 & 23.9 & 1574 & 10.7 & 15.5 & 34.4 \\
\hline Ferritin & $\mu \mathrm{g} / \mathrm{L}$ & 0.29 & Asia All & & & & & 1266 & 22 & 115 & 348 & 1606 & 3 & 23 & 124 \\
\hline & & & Japanese & & & & & 764 & 24 & 102 & 268 & 949 & 3 & 18 & 121 \\
\hline & & & Chinese & & & & & 265 & 28 & 152 & 442 & 332 & 3 & 28 & 158 \\
\hline & & & SE Asia & & & & & 191 & 39 & 162 & 499 & 282 & 5 & 38 & 159 \\
\hline EPO & $\mathrm{IU} / \mathrm{L}$ & 0.13 & Asia All & 2859 & 4.0 & 8.4 & 22.1 & 1269 & 3.8 & 7.7 & 15.8 & 1586 & 3.9 & 9.1 & 25.4 \\
\hline VitB12 & $\mathrm{ng} / \mathrm{L}$ & 0.18 & Asia All & 2869 & 135 & 347 & 779 & 1270 & 123 & 312 & 705 & 1599 & 152 & 377 & 821 \\
\hline Folate & $\mu \mathrm{g} / \mathrm{L}$ & 0.68 & Asia All & 2840 & 10.1 & 21.8 & 56.4 & 1262 & 9.1 & 19.5 & 44.6 & 1591 & 11.5 & 24.0 & 67.2 \\
\hline & & & Japanese & 1701 & 9.4 & 19.2 & 40.0 & 777 & 8.7 & 17.4 & 34.7 & 930 & 10.8 & 20.8 & 46.0 \\
\hline & & & Chinese & 599 & 12.6 & 28.3 & 72.8 & 267 & 11.8 & 24.6 & 58.4 & 332 & 14.1 & 31.6 & 82.2 \\
\hline & & & SE Asia & 477 & 13.3 & 29.7 & 72.5 & 195 & 12.0 & 25.5 & 61.1 & 282 & 14.5 & 33.5 & 74.2 \\
\hline AFP & $\mu \mathrm{g} / \mathrm{L}$ & 0.00 & Asia All & 2859 & 1.1 & 2.5 & 6.5 & 1271 & 1.2 & 2.7 & 6.8 & 1590 & 1.0 & 2.4 & 6.4 \\
\hline CEA & $\mu \mathrm{g} / \mathrm{L}$ & 0.10 & Asia All & 2880 & 0.4 & 1.3 & 4.1 & 1264 & 0.4 & 1.6 & 4.4 & 1606 & 0.4 & 1.1 & 3.4 \\
\hline CA19-9 & $\times 10^{3} \mathrm{IU} / \mathrm{L}$ & 0.14 & Asia All & 2887 & 0.8 & 6.4 & 30.0 & 1276 & 0.8 & 5.2 & 24.5 & 1608 & 0.9 & 7.6 & 33.3 \\
\hline CA125 & $\times 10^{3} \mathrm{IU} / \mathrm{L}$ & 0.00 & Asia All & & & & & 1267 & 3.2 & 7.5 & 16.2 & 1588 & 4.2 & 11.9 & 42.4 \\
\hline CA15-3 & $\times 10^{3} \mathrm{IU} / \mathrm{L}$ & 0.35 & Asia All & 2853 & 4.0 & 6.5 & 19.2 & 1276 & 4.0 & 7.0 & 18.8 & 1583 & 3.9 & 6.1 & 19.3 \\
\hline & & & Japanese & 1726 & 3.8 & 6.1 & 17.4 & 775 & 4.1 & 6.7 & 17.8 & 942 & 3.8 & 5.7 & 16.4 \\
\hline & & & Chinese & 592 & 4.2 & 6.9 & 20.4 & 266 & 4.5 & 7.3 & 19.6 & 331 & 3.9 & 6.5 & 20.0 \\
\hline & & & SE Asia & 478 & 4.0 & 8.3 & 19.9 & 196 & 4.4 & 9.2 & 21.3 & 284 & 3.8 & 7.6 & 20.4 \\
\hline PSA & $\mu g / L$ & 0.04 & Asia All & & & & & 1271 & 0.32 & 0.88 & 2.77 & 1596 & 0.000 & 0.005 & 0.038 \\
\hline PG1 & $\mu \mathrm{g} / \mathrm{L}$ & 0.06 & Asia All & 2834 & 22 & 42 & 85 & 1255 & 25 & 46 & 95 & 1584 & 22 & 39 & 8 \\
\hline PG2 & $\mu \mathrm{g} / \mathrm{L}$ & 0.00 & Asia All & 2842 & 4 & 8 & 29 & 1248 & 4 & 9 & 26 & 1585 & 4 & 8 & 3 \\
\hline PG1/PG2 & & 0.00 & Asia All & 2850 & 2.1 & 5.1 & 7.9 & 1260 & 2.0 & 5.3 & 8.1 & 1590 & 1.9 & 5.0 & 7. \\
\hline DHEA-S & $\mathrm{mmol} / \mathrm{L}$ & 0.00 & Asia All & & & & & 1273 & 2.4 & 6.1 & 12.1 & 1602 & 1.0 & 3.9 & 9. \\
\hline PRL & $\mu \mathrm{g} / \mathrm{L}$ & 0.04 & Asia All & 2862 & 4 & 10 & 29 & 1272 & 4 & 9 & 21 & 1591 & 5 & 12 & 3 \\
\hline $\mathrm{LH}$ & $\mathrm{IU} / \mathrm{L}$ & 0.00 & Asia All & & & & & 1272 & 1 & 3 & 7 & 1588 & 1 & 7 & 7 \\
\hline
\end{tabular}


(Table 3 Continued)

\begin{tabular}{|c|c|c|c|c|c|c|c|c|c|c|c|c|c|c|c|}
\hline \multicolumn{4}{|c|}{ International unit } & \multirow[t]{2}{*}{ n } & \multicolumn{3}{|c|}{ Male + Female } & \multirow[t]{2}{*}{$\mathbf{n}$} & \multicolumn{3}{|c|}{ Male } & \multirow[t]{2}{*}{ n } & \multicolumn{3}{|c|}{ Female } \\
\hline Analytes & Units & SDR-reg & Area & & LL & Me & UL & & LL & Me & UL & & LL & $\mathrm{Me}$ & UL \\
\hline$\overline{\mathrm{FSH}}$ & $\mathrm{IU} / \mathrm{L}$ & 0.00 & Asia All & & & & & 1263 & 2 & 5 & 14 & 1597 & 2 & 8 & 173 \\
\hline TSH & $\mathrm{mU} / \mathrm{L}$ & 0.16 & Asia All & 2872 & 0.4 & 1.4 & 4.0 & 1263 & 0.4 & 1.4 & 3.8 & 1599 & 0.4 & 1.5 & 3.9 \\
\hline FT4 & $\mathrm{pmol} / \mathrm{L}$ & 0.04 & Asia All & 2879 & 9.2 & 11.7 & 14.6 & 1273 & 9.4 & 11.9 & 14.9 & 1607 & 9.1 & 11.5 & 14.2 \\
\hline FT3 & $\mathrm{pmol} / \mathrm{L}$ & 0.00 & Asia All & 2872 & 38.6 & 4.56 & 5.50 & 1272 & 4.05 & 4.73 & 5.90 & 1596 & 3.80 & 4.43 & 5.31 \\
\hline $\operatorname{Tg}$ & $\mu \mathrm{g} / \mathrm{L}$ & 0.14 & Asia All & 2869 & 1 & 9 & 30 & 1248 & 2 & 9 & 30 & 1597 & 1 & 9 & 32 \\
\hline Insulin & $\mathrm{mIU} / \mathrm{L}$ & 0.24 & Asia All & 2858 & 1.8 & 4.6 & 11.8 & 1239 & 2.1 & 4.8 & 13.5 & 1599 & 1.9 & 4.4 & 10.8 \\
\hline \multirow[t]{4}{*}{ Adiponectin } & $\mathrm{mg} / \mathrm{L}$ & 0.36 & Asia All & 2861 & 3.7 & 9.5 & 22.8 & 1254 & 3.4 & 7.5 & 16.2 & 1590 & 4.7 & 11.5 & 24.0 \\
\hline & & & Japanese & 1296 & 4.2 & 10.9 & 23.6 & 467 & 3.7 & 8.3 & 17.2 & 824 & 5.7 & 12.7 & 25.6 \\
\hline & & & Chinese & 527 & 3.7 & 9.5 & 19.4 & 216 & 3.3 & 7.6 & 14.5 & 311 & 5.5 & 11.2 & 22.2 \\
\hline & & & SE Asia & 414 & 3.0 & 7.5 & 16.3 & 159 & 2.6 & 5.9 & 12.4 & 258 & 3.8 & 8.6 & 18.8 \\
\hline \multirow[t]{4}{*}{ PTH } & $\mathrm{ng} / \mathrm{L}$ & 0.41 & Asia All & 2866 & 21 & 45 & 92 & 1271 & 21 & 44 & 89 & 1598 & 21 & 45 & 97 \\
\hline & & & Japanese & 1720 & 23 & 48 & 96 & 779 & 23 & 48 & 93 & 943 & 24 & 49 & 101 \\
\hline & & & Chinese & 594 & 18 & 38 & 70 & 265 & 19 & 37 & 68 & 329 & 18 & 38 & 73 \\
\hline & & & SE Asia & 475 & 17 & 40 & 94 & 193 & 20 & 42 & 83 & 280 & 16 & 39 & 98 \\
\hline BoneALP & $\mu \mathrm{g} / \mathrm{L}$ & 0.00 & Asia All & 2869 & 6.4 & 11.4 & 20.6 & 1267 & 7.5 & 12.5 & 22.5 & 1601 & 6.2 & 10.5 & 19.1 \\
\hline \multirow[t]{4}{*}{ TRAP-5b } & $\mathrm{U} / \mathrm{L}$ & 0.30 & Asia All & 2814 & 0.8 & 1.8 & 3.7 & 1247 & 0.9 & 2.0 & 3.8 & 1563 & 0.7 & 1.6 & 3.5 \\
\hline & & & Japanese & 1676 & 0.8 & 1.9 & 4.0 & 764 & 1.0 & 2.1 & 4.2 & 915 & 0.8 & 1.7 & 3.8 \\
\hline & & & Chinese & 585 & 0.7 & 1.5 & 3.1 & 263 & 0.8 & 1.7 & 3.2 & 325 & 0.6 & 1.4 & 3.1 \\
\hline & & & SE Asia & 471 & 0.7 & 1.7 & 3.7 & 193 & 1.1 & 2.0 & 3.8 & 277 & 0.7 & 1.5 & 3.6 \\
\hline
\end{tabular}

When the standard deviation ratio due to region (SDR-reg) is 0.25 , the RIs are computed in four ways: from the entire data (Asia All), Japanese, Chinese, and Southeast Asian (including Ho Chi Minh City, Kuala Lumpur, and Jakarta) (SE Asia).

Table 4 Reagent manufacturers involved in the cross-comparison of results measured by immunoassays.

\begin{tabular}{lrrrrrrr}
\hline & Roche & Abbott & Tosoh & Siemens & BC & Fuji & Misc \\
\hline Ferritin & 6 & 8 & 2 & 1 & 1 & 1 & 5 \\
AFP & 10 & 9 & 2 & 3 & 2 & 3 & 2 \\
CEA & 10 & 9 & 2 & 3 & 2 & 3 & 2 \\
CA19-9 & 11 & 8 & 1 & 1 & 1 & 3 & 2 \\
CA125 & 12 & 9 & 1 & 1 & 0 & 3 & 2 \\
PSA & 11 & 10 & 1 & 0 & 2 & 1 & 2 \\
Estradiol & 7 & 5 & 2 & 2 & 0 & 0 & 1 \\
Progesterone & 3 & 3 & 1 & 1 & 0 & 0 & 0 \\
Testosterone & 3 & 2 & 2 & 2 & 0 & 0 & 0 \\
PRL & 5 & 7 & 3 & 2 & 2 & 0 & 1 \\
FSH & 5 & 8 & 3 & 2 & 2 & 0 & 1 \\
LH & 5 & 8 & 3 & 2 & 2 & 0 & 1 \\
TSH & 11 & 12 & 2 & 4 & 0 & 0 & 1 \\
FT4 & 11 & 12 & 2 & 4 & 0 & 0 & 1 \\
FT3 & 9 & 11 & 2 & 3 & 0 & 0 & 1 \\
Insulin & 6 & 6 & 6 & 2 & 1 & 2 & 1 \\
Cortisol & 3 & 0 & 3 & 1 & 3 & 0 & 2 \\
PTH & 7 & 0 & 1 & 3 & 0 & 0 & 0 \\
& 135 & 127 & 39 & 37 & 18 & 16 & 25 \\
\hline
\end{tabular}

Abbott, Abbott Diagnostics; BC, Beckman Coulter; Fuji, Fujirebio Diagnostics; Misc, miscellaneous; Roche, Roche Diagnostics;

Siemens, Siemens Healthcare Diagnostics; Tosoh, Tosoh Bioscience. those of Figure 2, and the other for the commercial QC specimens (red circle or cross). These results clearly indicate that commutable specimens such as freshly prepared sera from healthy volunteers are essential to make crosscomparisons of test results aimed at transferring RIs by the regression procedure.

\section{Discussion}

A multicenter study for derivation of RIs can be conducted by either of two schemes. One is by individual measurement by collaborating laboratories and integration of test results to derive common RIs. The other is by centralized measurement and derivation of RIs, followed by transference of RIs to the collaborating laboratories with or without cross-comparison of test results. The former scheme can be applied to a study that only targets standardized analytes. However, when non-standardized analytes are included, it is essential to apply the latter scheme. The present study is the first multicenter study of reference values to include non-standardized analytes measured by immunoassays. There was, however, some 

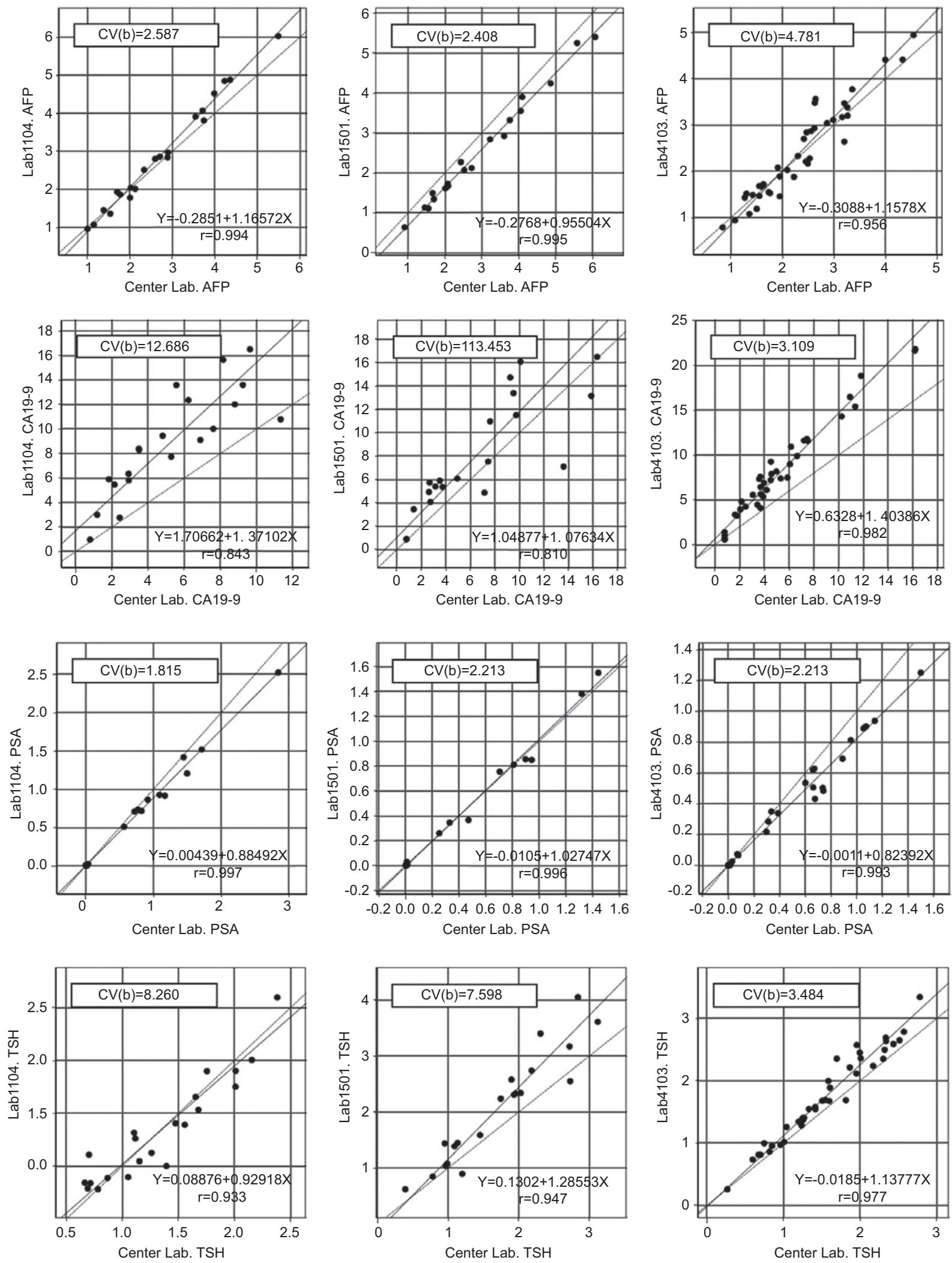

Figure 2 Cross-comparison of test results between the central and three local laboratories.

Typical results of cross-comparison of four non-standardized analytes (AFP, CA19-9, PSA, and TSH). The x-axis represents test results by the central laboratory, and the $y$-axis represents results obtained at the three local laboratories. The comparison was made in conventional unit. 


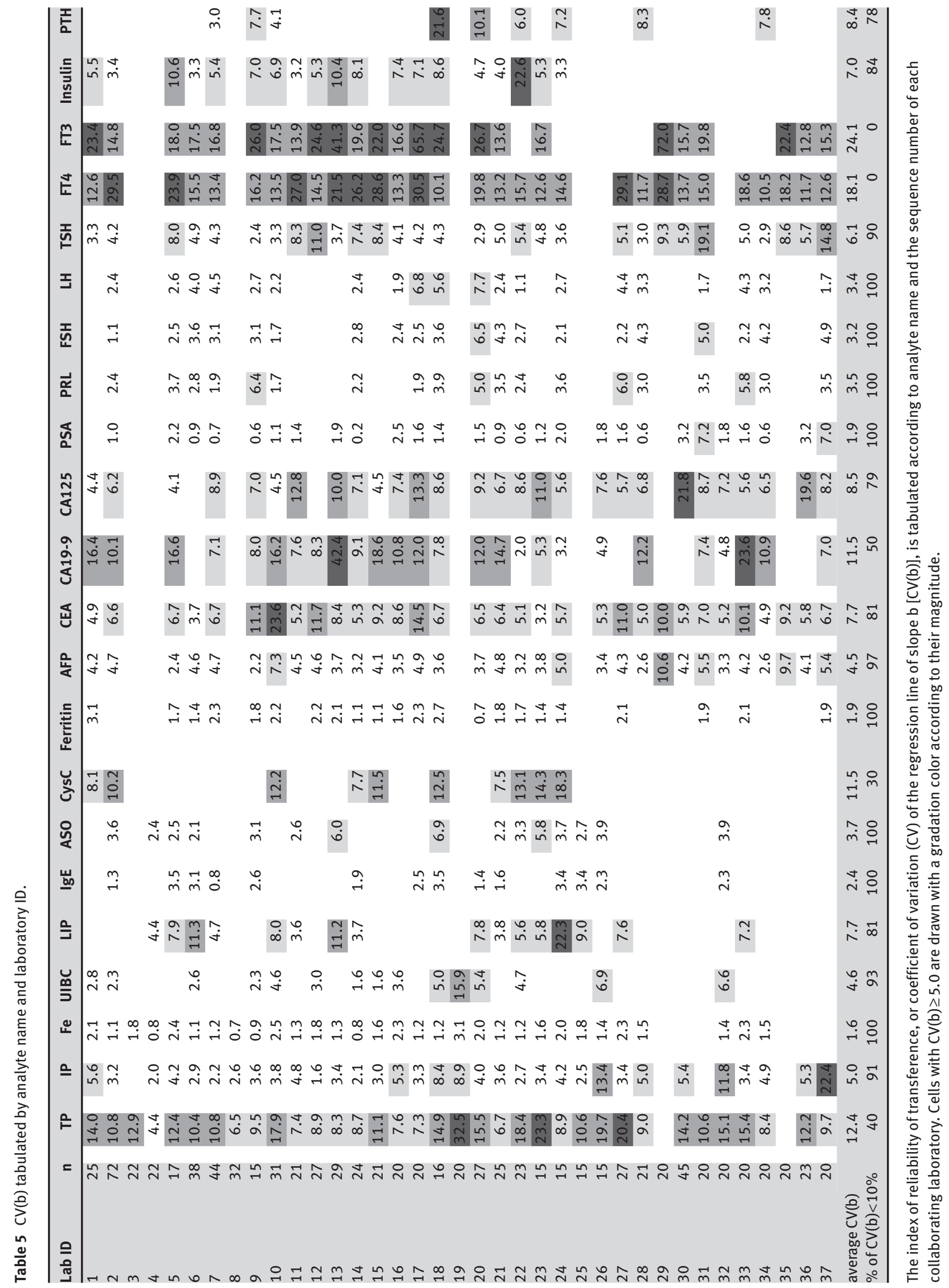



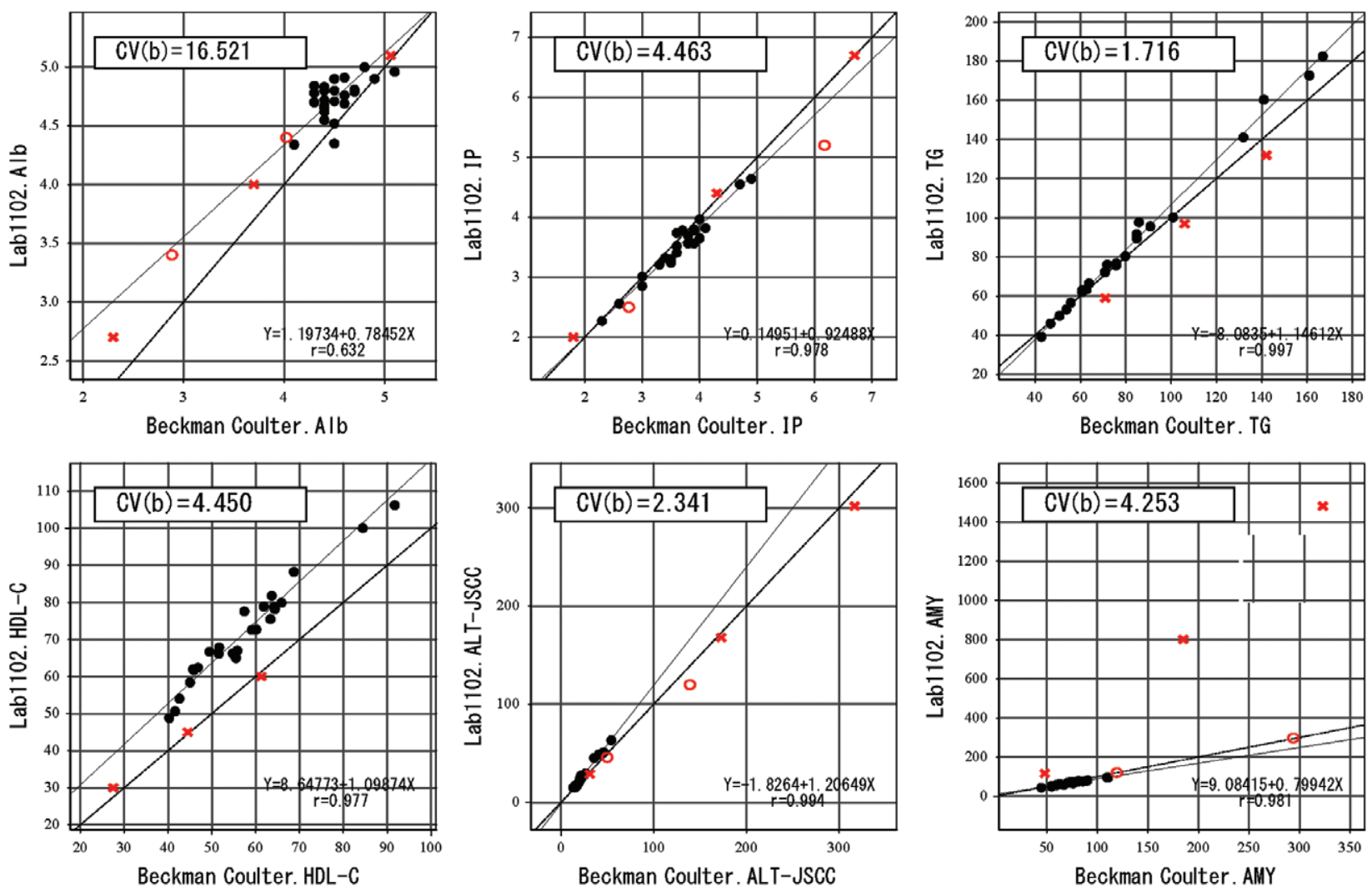

Figure 3 Commutability of commercial QC specimens in the cross-comparison.

Typical results of the cross-comparison plotted for two datasets: one for individual sera (black dots), as in those of Figure 1, and the other for the commercial QC specimens (red circle or cross, distinguishing the sources). The comparison was made in conventional unit.

hesitancy among would-be collaborating laboratories to take part in this study with no expected benefit unless the same reagents/platforms as those in the central laboratory were used. Therefore, it was needed to cross-compare test results between the central and local laboratories, and a service for transferring RIs to the participating laboratories based on the cross-comparison results by use of the reduced major axis regression was planned.

The precision of converting RIs depends primarily on the SE of the slope, SE(b), which in turn depends on both the correlation coefficient and the sample size used for the comparison, as expected from Equation (5) (also refer to Appendix 1). It is obvious that with increases in the data size $n$ and/or the correlation coefficient $r$, the precision of the conversion improves. For the standardized comparison, we chose to use $C V(b)$ by dividing it by the slope $b$.

Regarding the critical limit for providing the conversion service, it is difficult to set a fixed value that is suitable to all situations. This is because the precision depends not only on $n$ and $r$ described above but also on analytical variations and the range of the RI (LL-UL) relative to its median (see below). Therefore, we inspected all the crosscheck test results and checked for the behavior of $\mathrm{CV}(\mathrm{b})$ in relation to the data size and the degree of scatter around regression line. Then, we set $\mathrm{CV}(\mathrm{b})=10 \%$ empirically, as the critical value by consensus among the coordinators of the study. To demonstrate the performance of $\mathrm{CV}(\mathrm{b})$ and the implication its cut-off value, all pair-wise comparison made for four analytes are displayed in Supplementary Figure 2 as a reference. On the basis of the approximated formula, Equation (6), for the typical data size of $n=20$, 30 , and $40, \mathrm{CV}(\mathrm{b})=10 \%$ corresponds to $r=0.906,0.849$, and 0.787 , respectively, indicating that uncertainty of the conversion is equivalent for the three cases despite relatively large change in the $r$ value.

The number of specimens used for the comparison was in most cases $<40$, less than that described in the CLSI guideline (EP9-A2) entitled 'Method comparison and bias estimation using patient samples' [6]. Duplicate measurement as recommended in the guideline was also not done for the comparison. However, we obtained very low $\mathrm{CV}(\mathrm{b})$ of below $5 \%$ in most of the standardized analytes, a situation where we apparently do not need that many 
specimens for the comparison, with the notable exception of sodium $(\mathrm{Na})$, chloride $(\mathrm{Cl})$, potassium $(\mathrm{K})$, total calcium (Ca), and albumin (Alb), which showed CV(b) of $>10 \%$, as discussed below.

At any rate, we probably have to stratify the level of strictness in setting the acceptability criteria for the crosscomparison in the multicenter study. It varies from a situation where one just needs to confirm transferability of the RIs centrally determined to a situation where a regulatory group requests strict criteria for the transference. In fact, what we aimed at by the cross-comparison was to meet the need of the former situation, and thus, most laboratories were pleased with provision of the transference service.

However, we realized that it was very important not to measure all specimens for the cross-comparison in a single day. They should have been measured in two or more parts on separate days so that between-day variations of test results be considered in deriving the regression line.

As expected, the test results for the non-standardized analytes depended on reagent types and showed systematic bias between the central and local laboratories in the majority of cases. However, CV(b)s observed for the analytes mostly measured by immunoassays were as low as those for the standardized analytes, with $43 \%$ of them attaining a $\mathrm{CV}(\mathrm{b})$ of $<5 \%$. This observation should have great relevance in considering harmonization of test results of those analytes. In fact, an international forum called 'Improving Clinical Laboratory Testing through Harmonization' was held in October, 2010, in Gaithersburg, MD, USA, and the topic of harmonization of immunoassays has become an urgent global issue in the clinical chemistry societies [7]. It appears obvious that an indispensable step for harmonization is comparative measurement of a panel of commutable specimens or a set of multiple individual sera across reagents/platforms in the market. Then, the clinical chemical societies can find a way to build consensus among reagent manufacturers on how to align test results based on the comparison.

The large scatter around the regression line observed for TP, Alb, and the electrolytes (Supplementary Table 5) is mostly attributable to the narrow range (UL-LL) of each RI relative to the mid-point ((LL+UL)/2) of the RI, as was predicted by Linnet based on his simulation study to evaluate required number for samples for the method comparison [8]. This implies that a small change in the test result causes a large relative fluctuation in the cross-comparison using specimens limited to healthy individuals. Actually, the unit for reporting values of $\mathrm{Na}$ and $\mathrm{Cl}$ is $1 \mathrm{mmol} / \mathrm{L}$, and their RIs cover a range of only 6 units $(140-146 \mathrm{mmol} / \mathrm{L})$ and 8 units $(100-108 \mathrm{mmol} / \mathrm{L})$, respectively. Thus, there exists a large round-up error for the last digit in reporting test results: i.e., a difference of one unit $(1 \mathrm{mmol} / \mathrm{L})$ corresponds to $16.7 \%$ and $12.5 \%$ of the range of RIs for $\mathrm{Na}$ and $\mathrm{Cl}$, respectively. Similar situations exist for TP, Alb, K, and total Ca: a 1-unit change in the reported value makes a difference of $6.7 \%, 11.1 \%, 9.1 \%$, and $7.7 \%$ of the range of RIs, respectively.

From these theoretical considerations, the success of the transference of RIs on the basis of cross-comparison using specimens from healthy individuals depends on: 1) a wider range of the RI (UL-LL) relative to the median of the RI; 2) a larger sample size $n$ used for the comparison; and 3) a higher correlation coefficient $r$. It is also important to make a visual check for outlying data points which affect the regression line.

As for the regionality prominently observed for folate, we assume this will be attributable to the genetic differences in enzymes that are involved in processing folate in the body [9]. This is because supplementation of folate is not a common practice in East and Southeast Asia, even for women in the reproductive age. Actually, the pattern of regionality (higher values in Taiwan, Hong Kong, Ho Chi Minh City, and Jakarta) appears similar for both males and females. The possibility of higher consumption of green vegetables in those countries appears unlikely because regionality is still clearly observed even after adjusting for the level of vegetable consumption recorded in the questionnaire by multiple regression analysis (this finding will be reported in the third companion paper to be submitted in this series). In regard to the regionality observed for CA15-3, PTH, and adiponectin, we can only attribute them to genetic differences in concentrations of those proteins in blood.

Acknowledgments: This research was planned collaboratively by the: 1) Committee on Plasma Proteins (C-PP) and Committee on Reference Intervals and Decision Limits (C-RIDL) of the International Federation of Clinical Chemistry and Laboratory Medicine (IFCC); 2) the Scientific Committee of the Asia-Pacific Federation for Clinical Biochemistry (APFCB); 3) the Working Group on the Guideline for Common Reference Interval in the Japan Society of Laboratory Medicine (JSLM), and 4) the Committee on Plasma Protein of the Japan Society of Clinical Chemistry (JSCC). This study was also supported by the C-RIDL of the IFCC.

Research funds used included a Scientific Research Fund (No. 21406015: 2009-2011) provided by Japan Society for the Promotion of Science; a Research Promotion Project Fund of the JSLM (2008-2009); and a Scientific Research Fund of the APFCB. 
The clinical laboratories taking part in this cross-comparison study belong to the following institutions:

Outside Japan: 1) Gangnam Severance Hospital, Yonsei University, Seoul; 2) Kangbuk Samsung Hospital, Sungkyunkwan University, Seoul; 3) Myongji Hospital, Kwandong University College of Medicine, Gyeonggi-do; 4) Prince of Wales Hospital, Chinese University of Hong Kong, Hong Kong; 5) Macau Institute for Applied Research in Medicine and Health, Macau University of Science and Technology, Macau; 6) Mackay Memorial Hospital, Taipei; 7) Cathay General Hospital, Taipei; 8) Yuan Ching Clinical Laboratory, Taipei; 9) National Cheng Kung University, Tainan; 10) Chi-Mei Medical Center, Tainan; 11) National Heart Institute, Kuala Lumpur; 12) Gleneagles Intan Medical Centre, Kuala Lumpur; and 13) Medic-Lab, Ho Chi Minh City.

Inside Japan: 14) Keiyu Corp. Yoshida Hospital, Asahikawa; 15) Hokkaido University, Sapporo; 16) Kishimoto Clinical Laboratory, Tomakomai; 17) Iwate Medical School, Morioka; 18) Hirosaki University, Hirosaki; 19) Hachinohe Red-Cross Hospital, Hachinohe; 20) Hachinohe City Hospital, Hachinohe; 21) Chiba Cardiovascular Center, Ichihara; 22) Chiba University, Chiba; 23) Funabashi Municipal Medical Center, Funabashi; 24) Tokyo Medical and Dental University Chiba Hospital, Chiba; 25) Tokyo University,

\section{Appendix 1}

\section{Estimation of standard error of slope $b$ by the reduced major axis regression}

Assuming a dataset consisting of $n$ points of data pair ( $x$ and $y$ ), the reduced major axis regression is expressed as follows:

$$
y-\bar{y}=b(x-\bar{x})
$$

where $\bar{x}, \bar{y}$ represents the means of $x$ and $y$. The slope $b$ is derived as follows:

$$
b=\sqrt{\frac{S_{y y}}{S_{x x}}} \quad\left[S_{x x}=\sum_{i=1}^{n}\left(x_{i}-\bar{x}\right)^{2} S_{y y}=\sum_{i=1}^{n}\left(y_{i}-\bar{y}\right)^{2}\right]
$$

The mathematical method to derive standard error (SE) of $b, S E(b)$, is not known and commonly approximated by SE of slope $b^{\prime}, S E\left(b^{\prime}\right)$, by the ordinary leastsquare method by the following formula (references 2, 4).

$$
\begin{gathered}
y-\bar{y}=b^{\prime}(x-\bar{x}) \\
b^{\prime}=\frac{S_{x y}}{S_{x x}} \quad\left[S_{x y}=\sum_{i=1}^{n}\left(x_{i}-\bar{x}\right)\left(y_{i}-\bar{y}\right)\right]
\end{gathered}
$$

Tokyo; 26) Shinshu University, Matsumoto; 27) Yamanashi University, Kofu; 28) Nagoya University, Nagoya; 29) Anjo Kosei Hospital, Anjo; 30) Fujita Health University, Toyoake; 31) Osaka University, Suita; 32) Tenri Hospital, Tenri; 33) Osaka Municipal University, Osaka; 34) National Cardiovascular Center, Suita; 35) Kawasaki Medical School, Kurashiki; 36) Kurashiki Central Hospital, Kurashiki; 37) Okayama University, Okayama; 38) Okayama Medical Laboratory, Kurashiki; 39) Yamaguchi University, Ube; 40) Tokuyama Central Hospital, Shunan; 41) Yamaguchi Prefectural Medical Center, Hofu; 42) Saiseikai Yamaguchi Hospital, Yamaguchi; 43) Kochi Medical School, Nangoku; and 44) Ryukyu University Hospital, Naha.

\section{Conflict of interest statement}

Authors' conflict of interest disclosure: The authors stated that there are no conflicts of interest regarding the publication of this article.

Research funding: None declared.

Employment or leadership: None declared.

Honorarium: None declared.

Received June 29, 2012; accepted January 8, 2013

$$
S E(b) \simeq S E\left(b^{\prime}\right)=\frac{S}{\sqrt{S_{x x}}}
$$

where $s$ represents the standard deviation of data points around the ordinary least-square regression line. $s$ is derived as follows with $Y$ representing a predicted $y$ for a given $x$, or $Y=\bar{y}+b^{\prime}(x-\bar{x})$, and $r$ representing the correlation coefficient.

$$
\begin{aligned}
s & =\sqrt{\frac{\sum\left(y_{i}-Y_{i}\right)^{2}}{n-2}}=\sqrt{\frac{\sum\left(\left(y_{i}-\bar{y}\right)-b\left(x_{i}-\bar{x}\right)\right)^{2}}{n-2}} \\
& =\sqrt{\frac{\sum\left(y_{i}-\bar{y}\right)^{2}-2 b^{\prime} \sum\left(x_{i}-\bar{x}\right)\left(y_{i}-\bar{y}\right)+b^{\prime 2} \sum\left(x_{i}-\bar{x}\right)^{2}}{n-2}} \\
& =\sqrt{\frac{S_{y y}-2 b^{\prime} S_{x y}+b^{\prime 2} S_{x x}}{n-2}} \Leftarrow b^{\prime}=\frac{S_{x y}}{S_{x x}} \\
& =\sqrt{\frac{S_{y y}-S_{x y} b^{\prime}}{n-2}}=\sqrt{\frac{S_{y y}-S_{x y}^{2} / S_{x x}}{n-2}} \Leftarrow r=\frac{S_{x y}}{\sqrt{S_{x x} S_{y y}}} \\
& =\sqrt{\frac{\left(1-r^{2}\right) S_{y y}}{n-2}}
\end{aligned}
$$


Using the last formula, approximated $S E(b)$ can be expressed as follows.

$$
\begin{aligned}
& S E(b) \simeq S E\left(b^{\prime}\right)=\frac{S}{\sqrt{S_{x x}}}=\sqrt{\frac{\left(1-r^{2}\right) S_{y y}}{(n-2) S_{x x}}} \Leftarrow b=\sqrt{\frac{S_{y y}}{S_{x x}}} \\
& \quad=b \sqrt{\frac{1-r^{2}}{n-2}}
\end{aligned}
$$

\section{References}

1. Ichihara K, Ceriotti F, Tam TT, Sueyoshi S, Poon PM, Thonget ML, et al. The Asian project for collaborative derivation of reference intervals: (1) strategy and major resultsof standardized analytes. Clin Chem Lab Med 2013 Feb 23. [Epub ahead of print]. DOI: 10.1515/cclm-2012-0421.

2. Sokal RR, Rohlf FJ. Linear regression (Model II regression). In Biometry: the principles and practice of statistics in biological research, 3rd ed. San Francisco: W. H. Freeman, 1995:541-9.

3. McArdle BH. The structural relationship: regression in biology. Can J Zool 1988;66:2329-39.

4. Draper NR, Smith H. Applied regression analysis, 3rd ed. New York: John Wiley \& Sons, 1998.

5. Davison AC, Hinkley DV. Bootstrap methods and their application. Cambridge: Cambridge University Press, 1997.
Therefore, CV of slope $b$, or $C V(b)$, can be expressed as follows:

$$
C V(b)=\frac{S E(b)}{b} \times 100 \simeq 100 \sqrt{\frac{1-r^{2}}{n-2}}
$$

6. NCCLS. Method comparison and bias estimation using patient samples; approved guideline, 2nd ed. NCCLS document EP9-A2. Wayne, PA: Clinical and Laboratory Standards Institute, 2002.

7. Linnet K. Necessary sample size for method comparison studies based on regression analysis. Clin Chem 1999;45:882-94.

8. Miller GW, Myers GL, Gantzer ML, Kahn SE, Schönbrunner RE, Thienpont LM, et al. Roadmap for harmonization of clinical laboratory measurement procedures. Clin Chem 2011;57: 1108-17.

9. Ozarda Y, Sucu DK, Hizli B, Aslan D. Rate of T alleles and TT genotype at MTHFR 677C->T locus or C alleles and CC genotype at MTHFR 1298A->C locus among healthy subjects in Turkey: impact on homocysteine and folic acid status and reference intervals. Cell Biochem Funct 2009;27:568-77. 